\title{
Cladding-pumped Raman fiber with a W-type core designed for power scaling
}

\author{
J. Ji, C. A. Codemard, J. Nilsson \\ Optoelectronics Research Centre, University of Southampton, Southampton, SO17 1BJ, U. K. \\ E-mail:juj@orc.soton.ac.uk
}

Abstract: We design a cladding-pumped fiber Raman amplifier with a wavelength-filtering W-type core for the suppression of the $2^{\text {nd }}$-order Raman Stokes. This increases the useable inner-cladding-to-core area ratio, which benefits power scaling

Keywords: Frequency filter, Raman scattering, Raman laser

\section{INTRODUCTION}

Compared to their rare-earth (RE) doped counterpart, cladding-pumped (CP) fiber Raman devices [1]-[4] have their own concerns, such as the generation of the $2^{\text {nd }}$-order stimulated Raman scattering (SRS). Second-order SRS imposes a quite tight limitation on the inner-cladding-to-core area ratio within which pump power propagating in the inner cladding can be efficiently converted to $1^{\text {st }}$-Stokes power propagating in the core [5]. However, if the $2^{\text {nd }}$-Stokes can be suppressed, the limitation is relaxed and the inner-cladding area can be increased. A W-type fiber is a well-known short-wavelength pass filter [6] that have already been used for SRS-suppression in CP RE-doped fiber devices [7]-[8] by bending the fibers. The approach relies on the spectrally sharp bendloss characteristics of W-type fibers.

In this paper, we design a CP Raman fiber with a W-type core to suppress $2^{\text {nd }}$-order SRS. This way, we manage to increase the allowable area ratio from 8 to 36 and the inner-cladding diameter to $108 \mu \mathrm{m}$, thus allowing pump sources with lower brightness to be used. We consider a Raman pump wavelength of $1.55 \mu \mathrm{m}$, where suitable pump sources, e.g. pulsed Er-doped fiber lasers, are readily available and background loss is also low in silica fiber.

\section{RELAXATION OF AREA RATIO RESTRICTION}

Our analysis is based on the model presented in [5]. To achieve efficiently the desired conversion from the pump to the $1{ }^{\text {st- }}$ Stokes, there is a condition on area ratio of the inner-cladding to core area ratio [5] as shown below,

$$
\frac{G_{2}^{S R S}}{\zeta_{0}}=\frac{A_{\text {clad }}}{A_{\text {core }}}\left(\frac{\lambda_{0}}{\lambda_{2}}\right)=\left(\frac{D_{\text {clad }}}{d_{\text {core }}}\right)^{2}\left(\frac{\lambda_{0}}{\lambda_{2}}\right)<8
$$

where $G_{2}^{S R S}$ is the gain in nepers at the $2^{\text {nd }}$-Stokes and $\zeta_{0}$ is the nonlinear depletion of the pump induced by the $1^{\text {st }}$-Stokes. Furthermore, $\lambda_{0}$ and $\lambda_{2}$ are the wavelengths of the Raman pump and $2^{\text {nd }}$-Stokes, respectively, $A_{\text {core }}$ and $A_{\text {clad }}$ are the core and inner-cladding area, and $d_{\text {core }}$ and $D_{\text {clad }}$ are the core and inner-cladding diameter. Equation (1) follows from the symmetry of SRS in terms of Raman gain on the Stokes side and nonlinear depletion on the anti-Stokes side, and the effective-area dependence. See (11) and (12) in [5] for details. However, any loss at the $2^{\text {nd }}$-Stokes modifies (1) as below,

$$
\left(\frac{A_{\text {clad }}}{A_{\text {core }}}\right)_{\lambda_{i}}\left(\frac{\lambda_{0}}{\lambda_{2}}\right)\left(1-\frac{\alpha\left(\lambda_{i}\right) L}{\alpha\left(\lambda_{i}\right) L+16}\right)<8 \times \frac{g_{R}\left(\lambda_{1}, \lambda_{2}\right)}{g_{R}\left(\lambda_{1}, \lambda_{i}\right)}
$$

where $g_{R}\left(\lambda_{l}, \lambda_{i}\right)$ is the Raman gain coefficient at the wavelength $\lambda_{i}$ induced by power at the $1^{\text {st }}$-Stokes $\lambda_{l}, \alpha\left(\lambda_{i}\right)$ is the loss per unit length at $\lambda_{i}$, and $L$ is the fiber length. Note that while in the absence of filtering, the $2^{\text {nd }}$-order Raman Stokes will appear at the Raman gain peak (i.e., at $\lambda_{2}$ ), this is no longer necessarily the case with filtering since the filter will have some spectral dependence. Instead, the $2^{\text {nd }}$-order Raman Stokes typically appears at a shorter wavelength $\lambda_{i}$, such that $\lambda_{1}<\lambda_{i} \leq \lambda_{2}$, and all possible $2^{\text {nd }}$-order Stokes wavelengths need to be considered in (2).

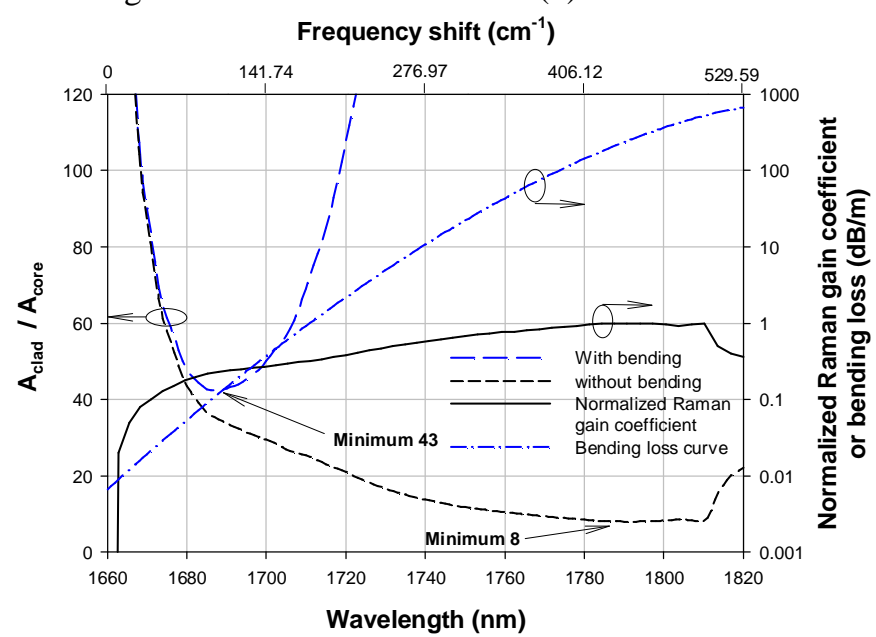

Fig. 1. $A_{\text {clad }} / A_{\text {core }}$ vs. wavelength and normalized Raman gain coefficient spectrum. The parameters of the W-type fiber: $A_{\text {core }}=36 \pi \mu \mathrm{m}^{2}$, cut-off wavelength is $1.85 \mu \mathrm{m}$ with bending radius of $20 \mathrm{~cm}$.

Figure 1 shows the spectrally resolved area ratio limit, with and without filtering, for a $30 \mathrm{~m}$ long fiber. Since the area ratio needs to be smaller than the spectrally resolved limit for all wavelengths, the overall area ratio limit is then the minimum of the spectrally resolved area ratio limit. The loss 
spectrum we assume is shown as well. $\lambda_{0}, \lambda_{1}$, and $\lambda_{2}$ are $1.55 \mu \mathrm{m}, 1.66 \mu \mathrm{m}$, and $1.79 \mu \mathrm{m}$, respectively. We see that indeed, the area ratio is limited at the peak of the Raman gain coefficient, at $1.79 \mu \mathrm{m}$, in the absence of filtering. With filtering, the limiting wavelength is shifted much closer to $\lambda_{l}$. At the same time, the overall area ratio limit improves from 8 without filtering to about 43 with filtering.

\section{W-TYPE FIBER DESIGN}

We still need to design a fiber to provide suitably sharp spectral filtering. For this, it helps to have the difference between the refractive index of the cladding $\left(n_{c l}\right)$ and the depressed region $\left(n_{d p}\right)$ as large as possible, although this is limited by the fabrication process for the low-loss silica fibers we require. The refractive index of the pure silica is 1.445 at $1.55 \mu \mathrm{m}$. We assume that the refractive index can be reduced to 1.44 , using the MCVD fabrication process. Therefore, $n_{d p}$ is set to 1.44 . Moreover, $n_{c l}$ is set at 1.457 , corresponding to a modest up-doping of the cladding (typically with Ge). The radius of the depressed region $\left(a_{d p}\right)$ is set as twice of the core radius $\left(a_{c o}\right)$ to provide flexible control of the fundamental cut-off wavelength $\left(\lambda_{c}\right)$ [9]. The core refractive index $n_{c o}$ and radius are then adjusted together to obtain specific values of $\lambda_{c}$. For each $\lambda_{c}$, the design that allows for the largest core area is selected as being the best one. This is typically the largest core that allows for sufficiently small $1^{\text {st }}$-Stokes bendloss at our maximum bend radius of $20 \mathrm{~cm}$.

In addition, different designs result in differently sharp bendloss spectra, and therefore in different area ratio limits. (This is a relatively small effect if $\lambda_{c}$ is kept constant.) We parameterize the bendloss spectrum using curve-fitting as shown in (3),

$$
\begin{aligned}
& \alpha(\Delta \lambda+\lambda)=\alpha\left(\lambda_{1}\right) \cdot \exp (p \cdot(\Delta \lambda+\lambda)+ \\
& p \cdot\left(-1.962 \cdot 10^{-3}+p \cdot 6.772 \cdot 10^{-3}\right) \cdot(\Delta \lambda+\lambda)^{2} \\
& \left.+p \cdot\left(-2.753 \cdot 10^{-6}+p \cdot 7.803 \cdot 10^{-6}\right) \cdot(\Delta \lambda+\lambda)^{3}\right)
\end{aligned}
$$

$\Delta \lambda$ is the wavelength of offset from $\lambda_{1}, \alpha\left(\lambda_{1}\right)$ is the loss at $\lambda_{1}$ $[\mathrm{dB} / \mathrm{m}]$.This results in a filter sharpness parameter $p$ which, close to $\lambda_{l}$, is the slope of the bendloss on a logarithmic scale with respect to wavelength. Thus, $p=0.02 \mathrm{~nm}^{-1}$ implies that the bendloss increases by a factor $e \approx 2.718$ over $50 \mathrm{~nm}$.

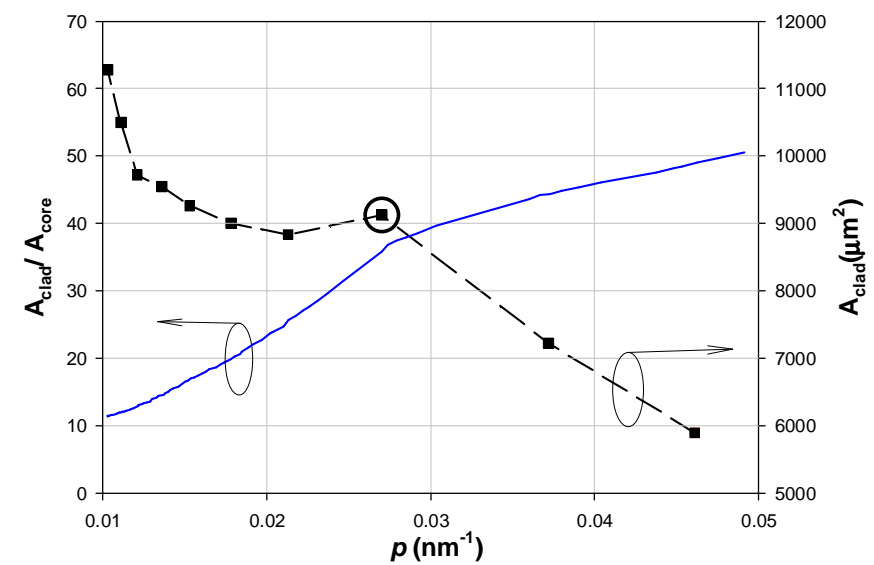

Fig. 2. Area ratio of the inner-cladding to the core and inner-cladding area $v s$. $p$ for W-type fiber.
Figure 2 shows how the permissible overall area ratio, determined from Eq. (2), as in Fig. 1, depends on $p$. The filter sharpness parameter $p$ is in turn determined from bendloss calculations according to Marcuse [10]. The loss at $\lambda_{I}$ is assumed to be $0.01 \mathrm{~dB} / \mathrm{m}$, leading to a total loss of $0.3 \mathrm{~dB}$ over the $30 \mathrm{~m}$ long fiber that we target. However, we have found that the influence of $\lambda_{I}$ and the fiber length on the overall area ratio limit is relatively small, provided that the total $1^{\text {st }}$-Stokes loss is fixed $(0.3 \mathrm{~dB}$ in our case).

While a larger area ratio is helpful, what really matters is a large inner-cladding area. This is also shown in Fig. 2, calculated as the overall area ratio limit multiplied by the core area of different fiber designs. Both a large area ratio and a large core area help to increase the allowable inner-cladding area. However, there is a trade-off between core area and filter sharpness, and thus area ratio. Thus, filter sharpnesses beyond $p=0.027 \mathrm{~nm}^{-1}$ require rapidly decreasing core sizes, to the detriment of the inner-cladding area. This is related to a hump in the Raman spectrum at around $80 \mathrm{~cm}^{-1}$ which reduces the benefits of filters sharper than $p=0.027 \mathrm{~nm}^{-1}$. For $p<0.027 \mathrm{~nm}^{-1}$, the allowable inner-cladding area first decreases and then increases again. This regime could also be interesting. However, at least with some fiber designs, this regime would be highly susceptible to micro-bending, which makes it less attractive. Therefore, we view the fiber with $p=$ $0.027 \mathrm{~nm}^{-1}$, and with $a_{c o}=9 \mu \mathrm{m}$ and $n_{c o}=1.4589$, as particularly promising. It is identified by a circle in Fig. 2.

Such designed fiber can be useful to generate high power or high energy source with diffraction-limited through CP fiber Raman amplifiers or lasers. More details, e.g., on optical damage which can also limit the area ratio, will be given at the conference.

\section{REFERENCES}

[1] J. Nilsson et al., "Cladding-pumped Raman amplifier", in $O A A$ (2002) Vancouver, Canada 14-17 Jul 2002 PD2.

[2] R. R. Rice, "Multimode Raman fiber amplifier and method", US patent no. $6,353,087,2002$.

[3] C. A. Codemard et al., "High-power continuous-wave cladding-pumped Raman fiber laser", Opt. Lett., Vol. 31, pp. 2290-2292, 2006.

[4] C. A. Codemard, J. K. Sahu, and J. Nilsson, "High-brightness pulsed cladding-pumped Raman fiber source at $1660 \mathrm{~nm}$ ", in Conf. Lasers and Electro-Optics /QELS 2007 Baltimore 6-11 May 2007, Paper CTuN3.

[5] J. Ji, C. A. Codemard, J. K. Sahu, M. Ibsen, and J. Nilsson, “Analysis of the conversion to the 1st Stokes in cladding-pumped fiber Raman amplifiers," Journal of Selected Topics in Quantum Electronics, Vol. 15, pp. 129-139, 2009.

[6] S. Kawakami, and S. Nishida, "Characteristics of a double clad optical fiber with a low-index inner cladding," IEEE J. Quantum Electron., vol. 10, pp. 879-887, Dec. 1974.

[7] D. B. S. Soh, et al., "Neodymium-doped cladding pump aluminosilicate fiber laser tunable in the $0.9 \mu \mathrm{m}$ wavelength rage," IEEE J. Quantum Electron., Vol. 40, pp. 1275-1282, 2004.

[8] J. Kim, P. Dupriez, C. Codemard, J. Nilsson, and J. K. Sahu, "Suppression of stimulated Raman scattering in a high power Yb-doped fiber amplifier using a W-type core with fundamental mode cut-off," Opt. Exp., Vol. 14, pp. 5103-5113, 2006.

[9] M. Monerie, "Propagation in doubly clad single-mode fibers," IEEE J. Quantum Electron., Vol. QE-18, pp. 535-542, 1982.

[10] D. Marcuse, "Influence of curvature on the losses of doubly clad fiber," Appl. Opt., Vol. 21, pp. 4208-4213, 1982. 